\title{
Varia: a tool for prediction, analysis and visualisation of variable genes
}

\author{
Gavin Mackenzie ${ }^{1}$, Rasmus W. Jensen ${ }^{2}$, Thomas Lavstsen ${ }^{2^{*}}$ and Thomas D. Otto ${ }^{1 *}$ (])
}

\section{*Correspondence:}

thomasl@sund.ku.dk;

thomasdan.otto@glasgow.

ac.uk

${ }^{1}$ Centre for Immunobiology,

Institute of Infection,

Immunity and Inflammation,

MVLS, University of Glasgow,

Glasgow, UK

${ }^{2}$ Department

of Immunology

and Microbiology,

Department of Infectious

Diseases, Centre

for Medical Parasitology,

University of Copenhagen,

1017 Rigshospitalet,

Copenhagen, Denmark

\begin{abstract}
Background: Parasites use polymorphic gene families to evade the immune system or interact with the host. Assessing the diversity and expression of such gene families in pathogens can inform on the repertoire or host interaction phenotypes of clinical relevance. However, obtaining the sequences and quantifying their expression is a challenge. In Plasmodium falciparum, the highly polymorphic var genes encode the major virulence protein, PfEMP1, which bind a range of human receptors through varying combinations of DBL and CIDR domains. Here we present a tool, Varia, to predict near full-length gene sequences and domain compositions of query genes from database genes sharing short sequence tags. Varia generates output through two complementary pipelines. Varia_VIP returns all putative gene sequences and domain compositions of the query gene from any partial sequence provided, thereby enabling experimental validation of specific genes of interest and detailed assessment of their putative domain structure. Varia_GEM accommodates rapid profiling of var gene expression in complex patient samples from DBLa expression sequence tags (EST), by computing a sample overall transcript profile stratified by PfEMP1 domain types.
\end{abstract}

Results: Varia_VIP was tested querying sequence tags from all DBL domain types using different search criteria. On average $92 \%$ of query tags had one or more $99 \%$ identical database hits, resulting in the full-length query gene sequence being identified (>99\% identical DNA $>80 \%$ of query gene) among the five most prominent database hits, for $\sim 33 \%$ of the query genes. Optimized Varia_GEM settings allowed correct prediction of $>90 \%$ of domains placed among the four most N-terminal domains, including the DBLa domain, and $>70 \%$ of C-terminal domains. With this accuracy, $\mathrm{N}$-terminal domains could be predicted for $>80 \%$ of queries, whereas prediction rates of C-terminal domains dropped with the distance from the DBLa from 70 to $40 \%$.

Conclusion: Prediction of var sequence and domain composition is possible from short sequence tags. Varia can be used to guide experimental validation of PfEMP1 sequences of interest and conduct high-throughput analysis of var type expression in patient samples. author(s) and the source, provide a link to the Creative Commons licence, and indicate if changes were made. The images or other third party material in this article are included in the article's Creative Commons licence, unless indicated otherwise in a credit line to the material. If material is not included in the article's Creative Commons licence and your intended use is not permitted by statutory regulation or exceeds the permitted use, you will need to obtain permission directly from the copyright holder. To view a copy of this licence, visit http:// creativecommons.org/licenses/by/4.0/. The Creative Commons Public Domain Dedication waiver (http://creativecommons.org/publi cdomain/zero/1.0/) applies to the data made available in this article, unless otherwise stated in a credit line to the data. 


\section{Background}

Pathogens can evade the immune system through polymorphic protein families interacting with host molecules. Examples of such gene families are the PIR and var in Plasmodium, the VSG in Trypanosoma brucei and the srs in Toxoplasma [1]. The most studied gene family is probably the Plasmodium falciparum var genes, which encode the $P$. falciparum membrane protein 1 (PfEMP1) family. PfEMP1 variants are inserted into the erythrocyte membrane to bind specific human endothelial receptors [2]. The PfEMP1 are targets of acquired immunity, and in response, the protein family has expanded and diversified to each parasite genome containing 60 copies of PfEMP1-encoding var genes of 6-12 kb [3-5]. The extracellular part of PfEMP1 (encoded by the var exon1) is composed of multiple DBL (Duffy binding like) and CIDR domains capable of binding specific human receptors. DBL and CIDR domains are classified into a few main domain types, DBL $\alpha-\zeta$ and CIDR $\alpha-\gamma$, which have been further divided into domain subtypes [4]. Due to varying sequence diversity homogeneity between main domain types, the stringency by which domain subtypes are defined differs between main domain types. A comprehensive description of PfEMP1 domain classification can be found in $[6,7]$.

The domain composition of each PfEMP1 can vary but follows a general pattern (Fig. 1), which includes an N-terminal NTS-DBL $\alpha$-CIDR "head structure" followed by varying combinations of domains in a semi-conserved order of main domain types. The exception to this rule is the highly conserved var2csa and var3 genes, which encode atypical domain compositions. The anchoring intracellular part of PfEMP1 is encoded by the var exon2.

Characterization of novel var genes and analysis of var gene expression in patient samples is difficult due to the diversity of the genes. Although it is possible to obtain

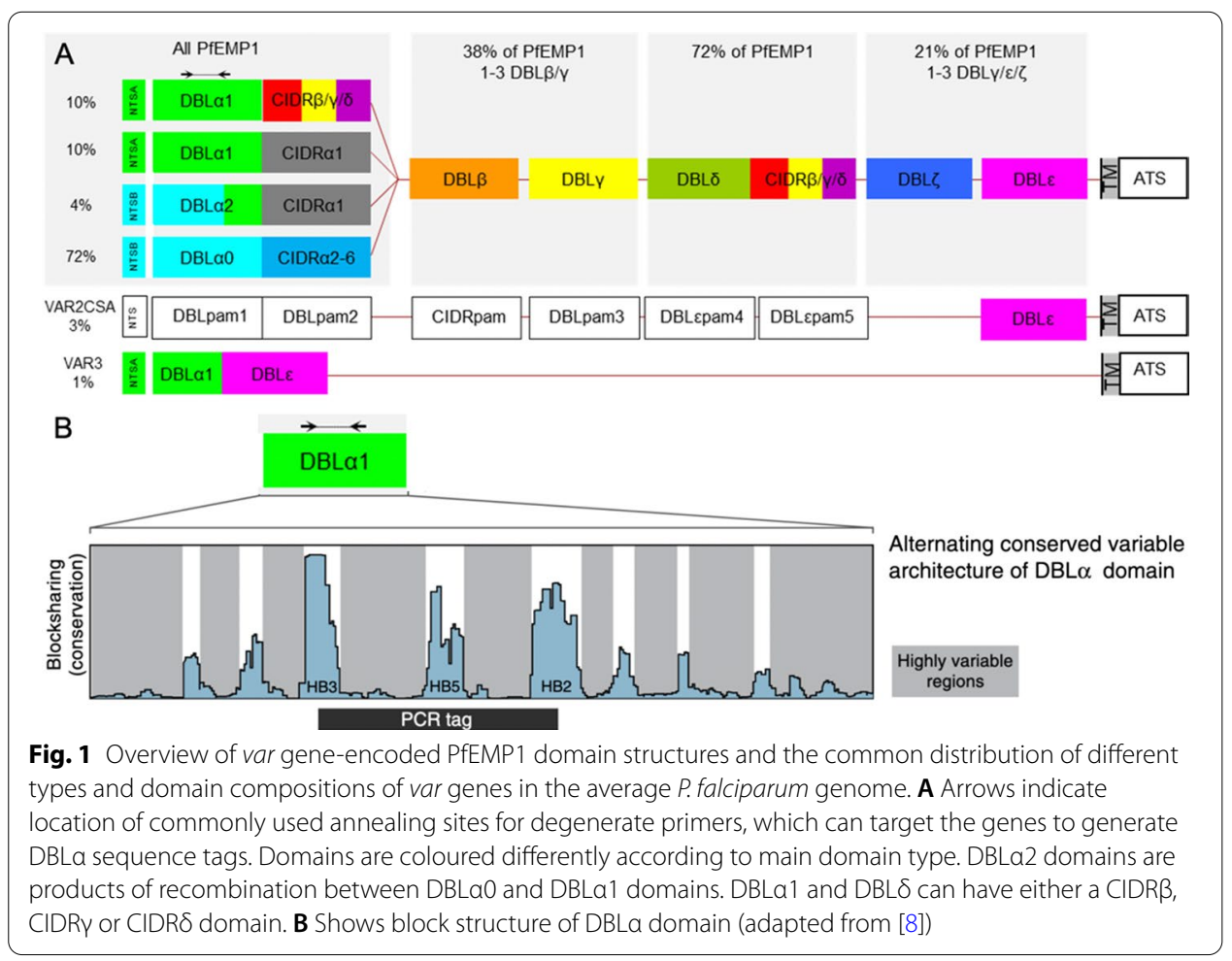


full-length var genes by assembling data from full genome sequencing, assembly and expression profiling from RNA sequencing is error prone, expensive and often challenging due to the small volumes of blood which can be drawn from severely ill children [9]. A cost-effective alternative has been to sequence reverse transcription (RT)-PCRamplified DBL $\alpha$ ESTs, and use these to quantify the relative expression level of different var specimens in a patient sample, such as in [10-13]. Analyses of diversity of DBL $\alpha$ tags amplified from gDNA are also employed to study dynamics of parasite populations [14].

Until now it has not been possible to infer the domain composition of the entire encoded PfEMP1 from short sequence tags. However, as the DNA sequence diversity in the DBL $\alpha$-tag region is extensive, it is possible that the flanking sequence of the originating genes can be predicted from the DBL $\alpha$-tag, if sufficient information on global sequence diversity is available.

Recently, through whole genome sequencing of 2400 parasites collected across the world, a database of over 140,000 var genes was generated [6]. We sought to exploit this unprecedented sequence depth and extend it with a further 750 samples to build and test a tool, which will enable reconstruction and experimental validation of the near fulllength variant genes from any sequence tag available. Specifically built for var genes, we extended the tool to also allow rapid quantification of PfEMP1 domain-specific expression in complex malaria patient samples by analysis of var DBL $\alpha$ expression tags.

\section{Implementation}

Varia offers two analysis pipelines (Fig. 2). In the Var Identification and Prediction (Varia_VIP) pipeline, the user provides one or more partial var sequences, which are used for searching a var database for near identical sequences. Hit sequences are clustered based on their full-length sequence similarity, and the domain composition of the longest gene sequence representing each cluster is visualized in circular plots and

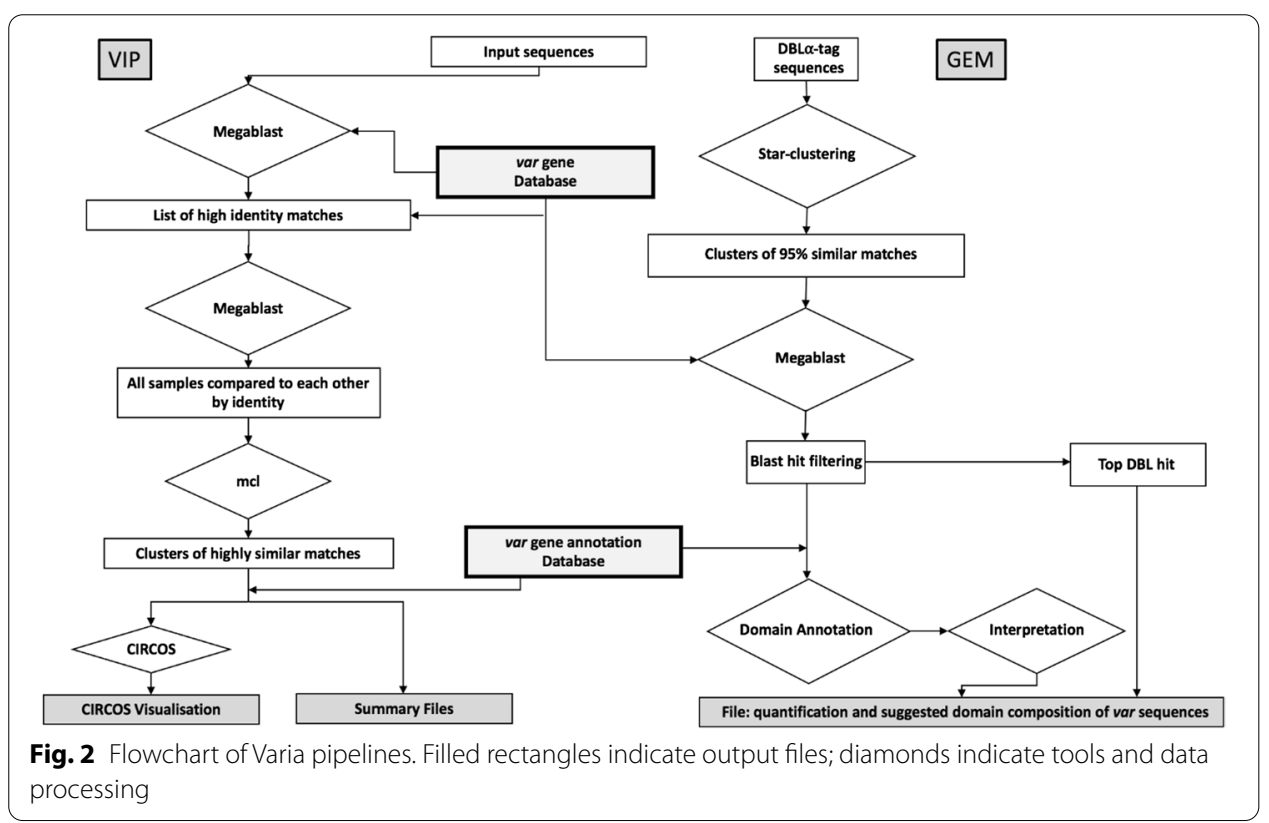


tabular output files (Additional file 1: Figures S1-S3). Note that the VIP module can be used on any gene family, if a reference database is provided.

In the Varia Gene Expression Module (Varia_GEM), batches of DBL $\alpha$ ESTs, such as those generated by high-throughput sequencing of multiple patient samples [11], are clustered to show the distribution of unique DBL $\alpha$-tag sequences in each data batch. For each unique DBL $\alpha$-tag, near identical sequences in the var database are identified and their domain composition processed to generate a single consensus prediction of the domain composition of each query gene, as well as the relative expression level of all known PfEMP1 domains in each batch of sequences. The output is given in Excel file format (Additional file 1), allowing subsequent statistical analyses of domain type association with e.g. clinical data pertaining to the samples.

For Varia, a new var genome database containing all assembled var gene contigs and their domain annotations was generated. The database includes 2400 Illumina sequenced parasite isolates (https://www.malariagen.net/projects/Pf3k and https://www.malar iagen.net/projects/p-falciparum-community-project) assembled in [6], 15 Pacific Bioscience sequenced $P$. falciparum clones $[3,6]$ as well as newly assembled var genes of $755 P$. falciparum isolates from the Community Project. The var genes of the 755 isolates were assembled using the var gene assembly pipeline as described in [6] and the sequences were added to https://github.com/ThomasDOtto/varDB/tree/master/Datasets/Addit ional755/. Not all genes from clinical isolates are full-length var genes, and in general, the exon 2 is missing from many of these genes. As we are interested in the prediction of sequences and domains, sequences shorter than $3 \mathrm{~kb}$ were excluded. We merged the new dataset with the existing, resulting in a total of 205,595 var gene sequences. From translated amino acid sequences, domain subtypes described in [4] were annotated using HMMer models developed in [6]. We used the command "hmmscan -cpu 12 noali -E 1e-6 -domE 1e-6" and parsed the results with an in-house Perl script. The Perl script and the HMMer models for the prediction are available at https://github.com/ ThomasDOtto/varDB/. As annotation was performed on complete and incomplete var sequences, these criteria may leave some sequence ends with incomplete domains unannotated.

To explore the var sequence prediction from this database, we randomly selected $40 \mathrm{k}$ DBL $\alpha$ sequences from the database. Specifically, we extracted the first 150 nucleotide bases starting from the LARSFADIG motif found in all DBL $\alpha$ domains. These sequences were blasted (megablast version 2.2.26, parameters: “-F F, E-value cutoff 1e-3") against four different partitions of the database: (1) the newly generated var sequences for this paper (41 k sequences), (2) the "normalised dataset" defined in [6] as the global representation of var gene diversity (720 genomes, 81 sequences, $92 \mathrm{k}$ sequences) (3) the full dataset analysed in [6] (2400 isolates, $162 \mathrm{k}$ sequences, labelled as Version 3) and 4) all the full compiled database (205,595 sequences; considered database version 4). A database sequence was considered a hit if it matched the DBL $\alpha$ query sequence with at least $99 \%$ identity over a 150 base pair overlap (self-hits were excluded). In addition to this, we analysed database hits using the full-length gene sequence of the same $40 \mathrm{k}$ query genes using megablast, as described. We counted database hits matching ( $>99 \%$ identity) the first 1000, 2000 and 3000 base pairs following the LARSFADIG motif and 
Table 1 Proportion of $40 \mathrm{k}$ var sequences of different length with identical sequence hits (>99\% identity) against databases of different size

\begin{tabular}{lllll}
\hline Hit length & \multicolumn{2}{l}{ Databases } & & \\
\cline { 2 - 5 } & $\mathbf{4 1} \mathbf{~ k}(\%)$ & $\mathbf{9 2} \mathbf{~ k}(\%)$ & $\mathbf{1 6 2} \mathbf{~ ( \% )}$ & $\mathbf{2 0 5} \mathbf{~ k}$ (\%) \\
\hline $150 \mathrm{bp}$ & 67.1 & 77.0 & 87.6 & 89.3 \\
$150 \mathrm{bp}$ (Africa) & 58.9 & 70.2 & 83.2 & 85.4 \\
$150 \mathrm{bp}$ (Asia) & 84.8 & 91.7 & 96.6 & 97.4 \\
$1 \mathrm{~kb}$ & 58.5 & 68.1 & 79.9 & 81.8 \\
$2 \mathrm{~kb}$ & 55.7 & 64.1 & 75.3 & 77.0 \\
$3 \mathrm{~kb}$ & 46.5 & 53.8 & 63.3 & 64.7 \\
full hits (80\%) & 30.6 & 41.8 & 53.4 & 55.7 \\
\hline
\end{tabular}

All sequences start at the LARSFADIG motif found in the N-terminal DBL domain (DBLa) of most var genes. Query sequences from African and Asian genes are shown separately for the 150 bp sequences

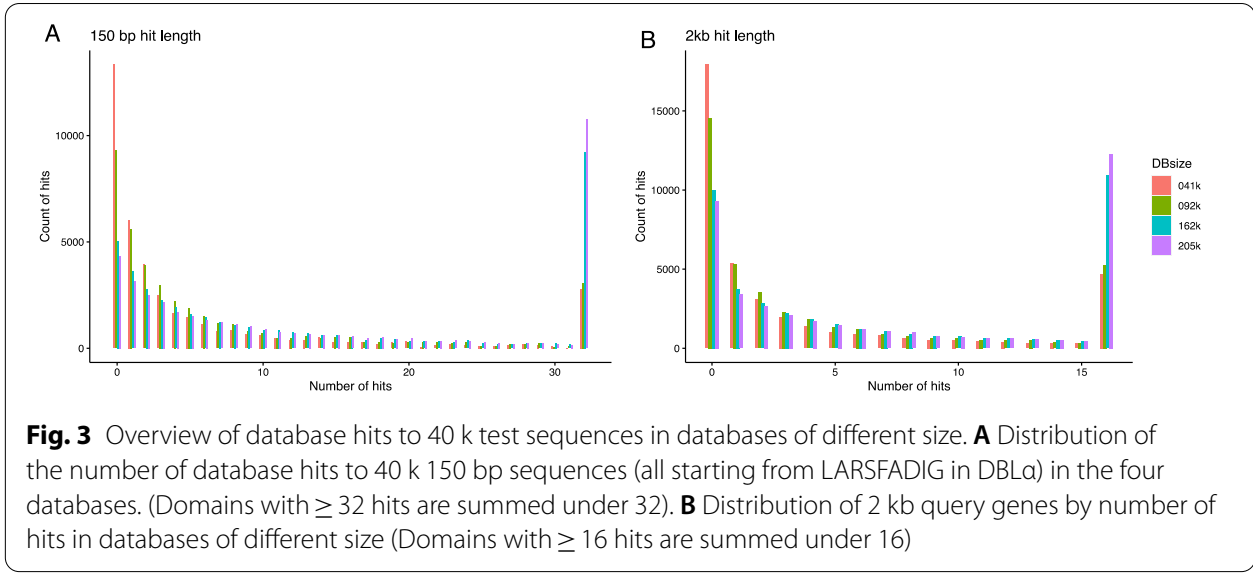

hits matching $>80 \%$ of the length of the complete input sequence (Table 1 and Fig. 3). The two query files can be found in the GitHub repository within the SimilarityTests directory.

\section{Results}

First, we evaluated the opportunities we can achieve with Varia. As expected, increasing the database size resulted in an increased proportion of query genes with database matches and more hits per query gene. However, even with the largest database, more than $10 \%$ of 150 bp query sequences are without any hit. The var gene database is biased towards genomes from South East Asian parasite isolates [6], and larger diversity has been reported among var genes of African isolates compared to Asian isolates. Separating the $40 \mathrm{k} 150 \mathrm{bp}$ query sequences by African or Asian origin showed that Asian sequences were more likely to have a database match (97\%) than African sequences (85\%). The hit rate drops with the length of query sequence, and for full-length sequences just over $50 \%$ of the sequences have complete matching hits. Increasing the database size from 160 to $220 \mathrm{k}$ makes little difference, but larger databases increase the hit frequency (Fig. 3). The most marked drop is seen when changing hit lengths from 2 to $3 \mathrm{~kb}$, which resulted in hit rates going from $\sim 77 \%$ to $\sim 65 \%$. This can be explained by 
the recombinogenic nature of var genes and the presence of a major recombination hotspot identified mid-var at 2-3 kb following the N-terminal DBL $\alpha$-CIDR domain complex encoded in most PfEMP1 [4].

In practice, var tags are likely to be generated from sequencing of PCR-amplified fragments spanning across variable regions roughly corresponding to the second structural subdomain of DBL domains [4]. The varying sequence diversity homogeneity between main types of DBL domains is likely to affect the ability of Varia_VIP to predict correct domain compositions. To assess this, Varia_VIP predictions were made from DBL tag sequences corresponding to the second structural subdomain [4] of all main DBL domains found in var genes from the 15 long-read sequenced and annotated $P$. falciparum genomes. A total of 971 DBL $\alpha-\zeta$ domain tags were run through Varia_VIP, excluding the test sequences from the database (Table 2).

This showed that tags from DBL $\alpha, \beta, \delta$ and $\gamma$ domains resulted in fewer different hit sequences (grouped into clusters of identical hit sequences) and more frequent correct annotations compared to DBL $\varepsilon$ and DBL $\zeta$ domains. This observation can be explained by the differences in the distribution of diversity within these main domain types. DBL $\alpha$, $\beta, \delta$ and $\gamma$ domains are highly diverse in sequence and diversity is homogeneous. Conversely, DBL $\varepsilon$ and $\mathrm{DBL} \zeta$ domain sequences distribute into distinctly different groups of highly similar sequences. Thus, as identical DBLe and DBL $\zeta$ domains are found in many different PfEMP1 variants, correct predictions are difficult to make from these domain tags.

As expected, lowering the similarity threshold increased the proportion of queries with correctly predicted annotations but at the cost of a higher number of different predictions. Specifically, for the DBL $\alpha$ domain tags, which are found in the $5^{\prime}$-end of all var genes, a correct annotation was identified for $72 \%$ of the query genes at $99 \%$ sequence similarity threshold. The correct annotation was found among 14 suggested predictions, on average. At this $99 \%$ similarity threshold, prediction of the exact query DNA sequence, defined as $99 \%$ identity over at least $80 \%$ of the sequence, was successful for $29 \%$ of the DBL $\alpha$ domain tags. Lowering the similarity threshold to $90 \%$ resulted in a limited increase to $78 \%$ of the query genes with correct annotation among the suggested domain compositions, and an increase in the number of alternative suggestions. For this reason, we recommend applying the highest similarity threshold resulting in database hits when using Varia_VIP to search for putative domain compositions and sequences of query genes, to allow manageable experimental validation (e.g. by PCR) of the query sequence.

As noted above, the current var gene database is biased towards genomes from South East Asian parasite isolates [6]. At $95 \%$ sequence similarity over 200 base pairs, $98 \%$ of DBL $\alpha$ tags from African isolates had one or more database hits, whereas this was the case for $100 \%$ of DBL $\alpha$ tags from Asian isolates. This resulted in a correctly annotated cluster-representative sequence by Varia_VIP (any cluster) for 60\% versus $85 \%$ of the query tags from African vs. Asian isolates, respectively.

In some studies, for example of var gene expression in patients, a rapid prediction of the single most likely PfEMP1 domain composition of a large number of sequences is required. For this, we built Varia_GEM, which, based on all hit sequences to a DBL $\alpha$ EST, returns the likely consensus domain annotation of the query gene. As the ability 


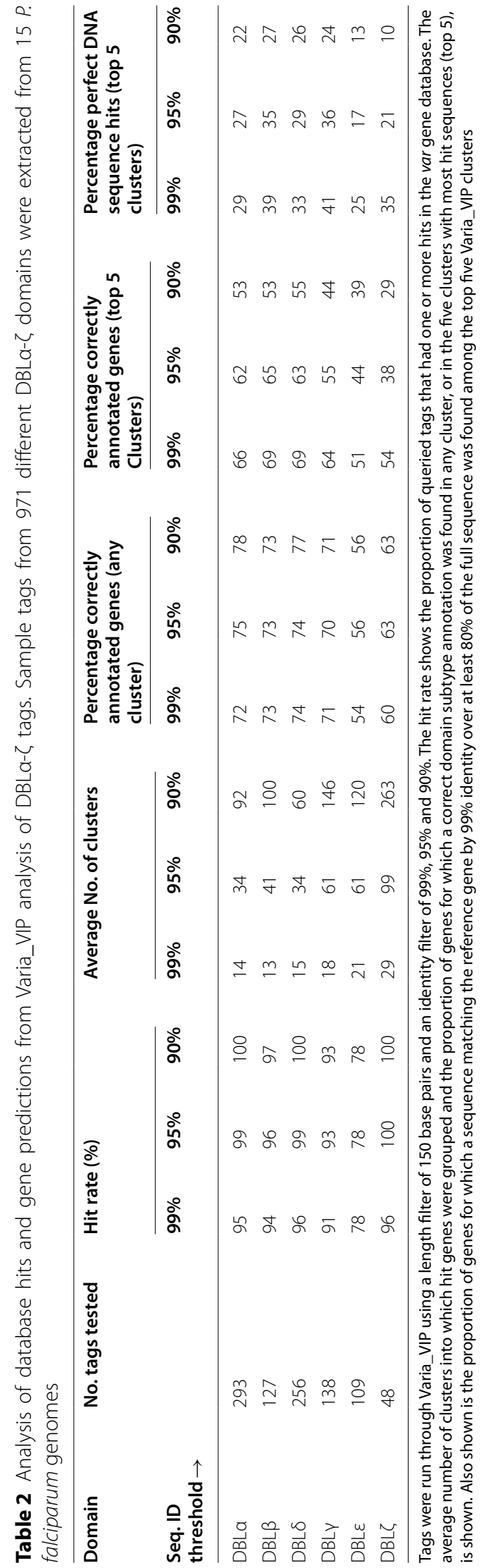


to predict correct annotations depends on domain type and distance to the query tag, Varia_GEM was designed to assess domain annotation for each domain position relative to the PfEMP1 N-terminal. For each domain position (D1-D10; DBL $\alpha$ is always at position D2) the tool determines if a specific PfEMP1 main domain type or domain subtype is dominant (>66\%) among hit gene sequences. If this is the case, the tool returns the consensus annotation in a tabular format, along with quantitative data of frequency of ESTs associated with the domain or domain composition, to allow quantitative analysis of PfEMP1 domain traits with e.g. clinical features pertaining to the origin of the sequences.

To determine parameters for optimal domain type prediction by Varia_GEM, we tested main domain type and domain subtype predictions at different identity threshold values. We extracted 220 randomly selected DBL $\alpha 0,1$ and 2 tag sequences (660 in total) from the var gene database, as they would be amplified by the commonly used DBL $\alpha$ EST primers [15]. The cognate genes were removed from the database, and the database was searched for genes sharing $99,97,95,93 \%$ and $85 \%$ identity across 200 base pairs of the DBL $\alpha$-tags. This resulted in an average $\sim 25$ hits per DBL $\alpha$ tag at $99 \%$ threshold, increasing to 44 hits at $93 \%$ and 114 hits at $85 \%$ (Fig. $4 \mathrm{~A}$ ). The consensus domain composition generated for each DBL $\alpha$-tag was compared to the known domain composition of the gene to calculate the accuracy of the predictions at each position of the domain (Fig. 4B). The accuracy was calculated both at the main domain type and domain subtype level, as follows:

- Average main domain accuracy: An annotation was considered incorrect if the predicted main domain type was wrong, or if a non-existing domain was predicted (i.e. a domain was predicted at a position where no domain was present in the original sequence).

- Average domain subtype accuracy: An annotation was considered incorrect if the predicted domain subtype was wrong, or if a non-existing domain was predicted (i.e. a domain was predicted at a position where no domain was present in the original sequence).

Next, the frequency by which the tool could predict the correct var gene domain compositions (coverage) was calculated for each domain position.

- Average coverage (main domain type): The proportion of genes with a correctly predicted main domain type at said position. A domain annotation was considered incorrect if a wrong main domain type was predicted, or a domain annotation was missing (i.e. no domain type was predicted where a domain was present in the original sequence).

Lowering the blast identity threshold and thereby including a higher number of hits resulted in fewer predictions and fewer correct domain annotations (Fig. 4B). However, main domain predictions were robust, with $85 \%$ accuracy over the first four domains. Lowering the blast identity threshold from 99 to $93 \%$ caused a slight decrease in main type accuracy, but a moderate decrease in coverage and domain subtype accuracy. Lowering the identity threshold further to $85 \%$ caused a significant 


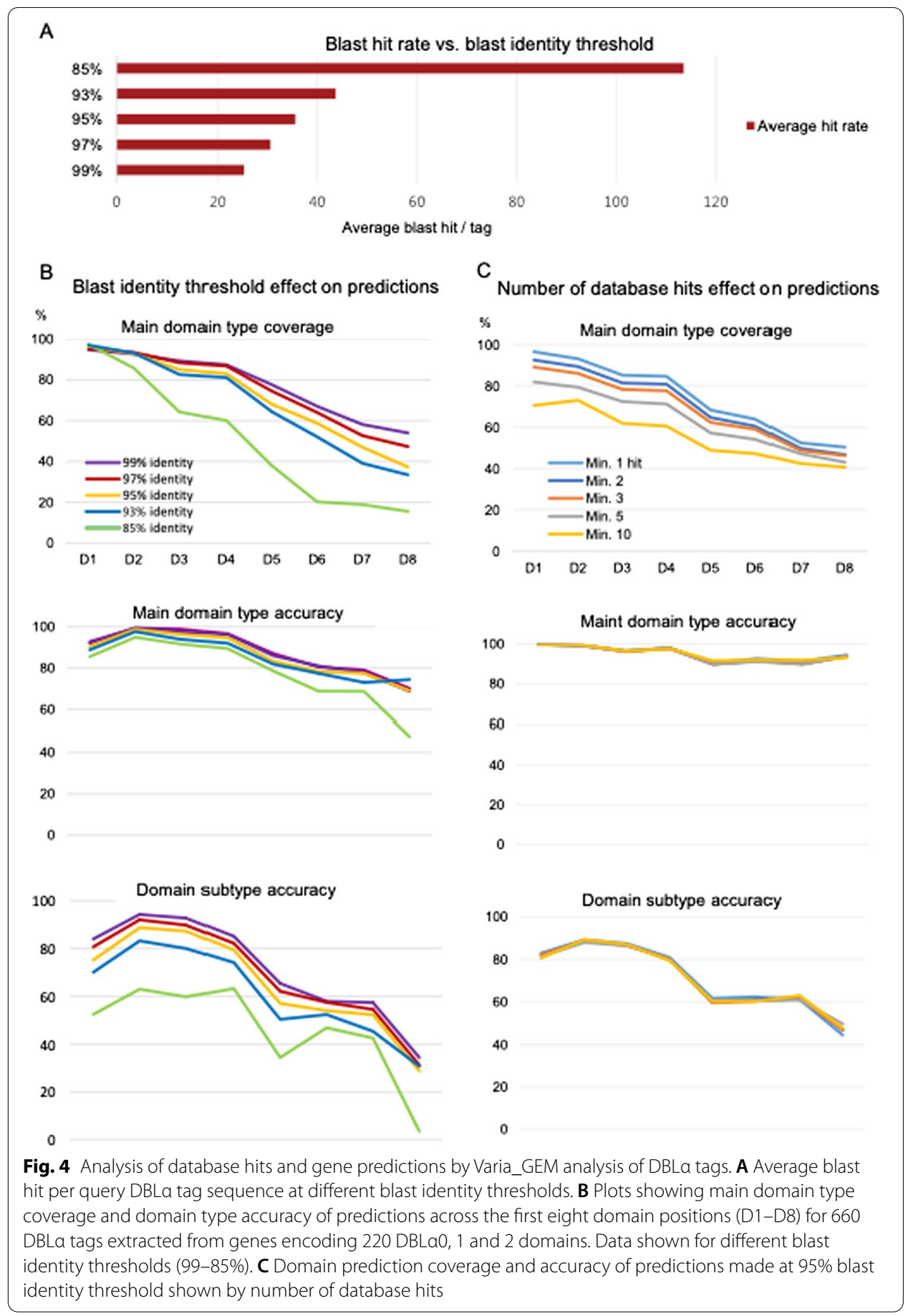

drop in main domain coverage and domain subtype accuracy, but again only a small decrease in main domain type accuracy. The most significant effects from lowering the blast identity threshold, was seen on domains at position D5-8. The level of coverage and accuracy was similar for different subtypes of DBL $\alpha$ (DBL 0,1 and 2, not shown). 
Most frequent and correct domain predictions were generated using the $99 \%$ blast identity threshold. This threshold is likely to be too stringent for analysis of sequencing data, which may contain various minor sequence errors. Instead, a 95\% threshold was chosen as default for Varia_GEM. This threshold results in a $0-10 \%$ drop in main domain type coverage and domain subtype accuracy, as well as a $0-3 \%$ decrease in main domain type accuracy, on average. Using the $95 \%$ identity threshold, the effect of the number of blast hits on domain prediction was investigated (Fig. 4C). This showed that a higher number of hits adversely affected the coverage but did not affect the accuracy of the predictions, and that including predictions made based on only one hit sequence resulted in most frequent correct predictions. Based on this, Varia_GEM was set to predict on every tag regardless of the number of database hits.

\section{Discussion}

Prediction of variant genes from short sequence tags is challenged by the speed and molecular mechanisms by which the genes evolve. Here, we empirically explored to which degree we can predict $P$. falciparum var genes from short DNA sequence tags. Previous studies have shown that the extensive diversity of the var genes is ancient and in large depends on recombination [6]. This challenges prediction of var genes. Moreover, prediction is hampered by an ambiguous clustering of PfEMP1 domains into specific subtypes. Main domain types (DBL $\alpha-\zeta$, and CIDR $\alpha-\gamma$ ) are well defined (Figs. 3B, 4), but the sequence diversity homogeneity differs between main domain types, and domain subtyping is uncertain $[4,6]$ and possibly reflects antigenic rather than functional diversification [18]. For example, found at domain position 3, CIDR $\alpha$ domain sequences separate distinctly into two groups, CIDR $\alpha 1$ or CIDR $\alpha 2-6$ domains, but the 18 subsets of CIDR $\alpha 2-6$ domains, all expected to bind CD36, are less well segregated and defined. The recombinogenic nature of var genes makes it likely that gene and domain predictions will be most accurate nearest to the analyzed tag and most uncertain across sites of frequent recombination. This explains the drop in main domain coverage and domain subtype accuracy with domain position, and the significant drop in correct predictions around domain position D5 (Table 1), which corresponds to a major recombination hotspot found mid-var gene [4]. However, the high overall main domain accuracy of Varia predictions across all domain positions shows that if a main domain type is predicted, this is very likely to be true.

As an example of Varia_VIP use, we predicted the full var gene sequences from DBL $\alpha$ tags PCR-amplified in [16]. In this study, the authors experimentally validated five fulllength var genes from the tags. Figure 5A shows the different full-length genes Varia suggests are the origin of a PCR-amplified DBL $\alpha$ tag for two of the DBL $\alpha$ tags (Complete results in Additional file 1: Table S1). The Circos plot visualizes the options to design primers to experimentally verify the var gene from a few potential sequences. Overall, Varia found the full sequences in the first two clusters, see Additional file 1. As an example of Varia_GEM use, Fig. 5B shows a section of the output file of the predicted var gene expression profile of one of the 32 P. falciparum-infected adult travellers returning to Germany [17]. A comprehensive comparison of the RNA-seq and DBL $\alpha$-EST and Varia GEM predicted var expression profiles are given in [17]. In brief, a partial domain annotation was made for $\sim 85 \%$ of DBL $\alpha$-ESTs; $~ 83 \%$ of all unique DBL $\alpha$-ESTs were found in the RNA-seq approach, and $82 \%$ of the most abundant transcripts encoding a DBL $\alpha$-tag 


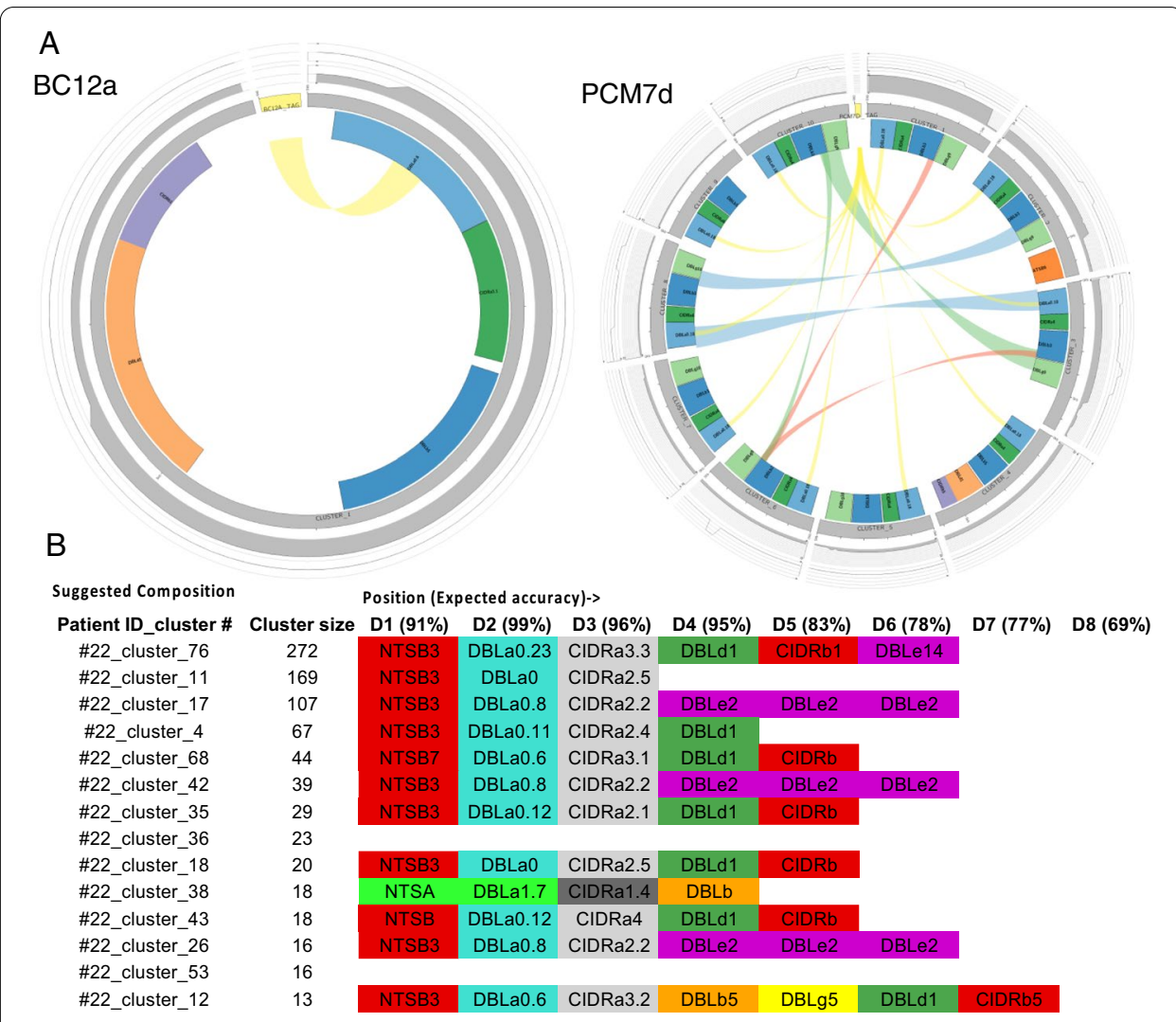

Fig. 5 Examples of Varia use and output. A Circos plot of domain-annotated full-length var genes suggested by Varia_VIP to represent the full length sequence from a DBLa-EST analysis of [16]. The BC12a tag returns one and the PCMd7 tag returns nine different sequence clusters. The format of plots is detailed in the Additional file 1, but in short, each segment represents a sequence cluster from a single patient. The coloured boxes are the domain annotations, and the coloured ribbons between domains are blast hits between the suggested sequences (the colour depends on the colour of the domain. The yellow box is the input sequence). For each cluster, the blast hit to other gene clusters (outer graph, correlates with blast ribbons) and the number of hits within the cluster (bar plot) is shown. B Section of the Varia_GEM output file generated from DBLa ESTs amplified from patient number 22 in a recent study of P. falciparum infected travelers [17]. Identical DBLa_ESTs are clustered, and the read counts represent the relative expression level of each specific DBLa EST tag. Here, DBLa EST sequence cluster number 76 was the most prominently expressed gene. The Varia_ GEM-predicted domain composition of the full-length transcripts is shown for each DBLa EST. The output file also includes these data stratified per domain type such that the overall relative expression level of e.g. CIDRa1 domains can be assessed for each single patient and all patients in a dataset. The expected accuracy of main domain type prediction at each domain position based on the current database is shown

region (upper 75th percentile of RNA-seq contigs) were also found by the DBL $\alpha$-tag approach.

\section{Conclusions}

Due to the diversity of PfEMP1 sequences, resolution of unknown full-length var gene sequences and their domain structure has required cumbersome laboratory proceedings. With Varia, we generated a tool that predicts var gene sequences from small easily obtained sequence fragments, to allow the community to reconstruct genes of interest. The likelihood of correct predictions of sequence and domains flanking the query tags was high, whereas predictions distant to the query tag were less likely. These limitations are caused by the sequence diversity and recombination history of 
the var genes. Improved rates of prediction may be achieved if additional P. falciparum genomes, in particular from Africa or South America, are added to the var gene database. However, with awareness of these limitations, sequences and domain compositions of var genes of interest can be experimentally validated by PCR, and differential var type expression between groups of patient samples can be assessed. The Varia tool will thus be useful to understand the distribution and clinical importance of different var gene subsets, and can be adapted to predict sequence and domain compositions from tags of other variable gene families.

\section{Availability and requirements}

Project name: VARIA,

Project home page: https://github.com/GCJMackenzie/Varia,

Operating system(s): Linux (Possible to use through Virtual box for Max and Windows), Programming language: Bash and Python,

Other requirements: Database can be downloaded from https://github.com/Thoma sDOtto/varDB, as indicated on VARIA GitHub page. Varia builds on several basic bioinformatics tools, like NCBI BLAST, mcl, Circos and samtools.

License: GNU General Public License v3.0,

Any restrictions to use by non-academics: no restrictions.

Abbreviations

EST: Expression sequence tags; CIDR: Cysteine-rich inter domain region; DBL: Duffy binding-like; PfEMP1: P. falciparum Membrane protein 1.

\section{Supplementary Information}

The online version contains supplementary material available at https://doi.org/10.1186/s12859-022-04573-6.

Additional file 1: Information on implementation and usage of Varia. Examples of output.

\section{Acknowledgements}

We thank MalariaGEN Plasmodium falciparum Community Project to allow the use sequencing information of clinical isolates.

Authors' contributions

The study was conceived by TO and TL. The software development and testing were carried out by GM and RWJ with input about functionality from TO and TL. TO generated the var database. TL and TO wrote the paper. All authors read and approved the final manuscript.

Funding

TO is supported by the Wellcome Trust grant 104111/Z/14/ZR. RWJ and TL was supported by the Lundbeck Foundation. Funding bodies had no role in the study.

Availability of data and materials

All data can be found at https://github.com/GCJMackenzie/Varia. The database with the var gene sequences can be found at https://github.com/ThomasDOtto/varDB.

\section{Declarations}

Ethics approval and consent to participate Not applicable.

Consent for publication

Not applicable.

Competing interests

The authors declare that they have no competing interests. 
Received: 12 March 2021 Accepted: 10 January 2022

Published online: 24 January 2022

\section{References}

1. Reid AJ. Large, rapidly evolving gene families are at the forefront of host-parasite interactions in Apicomplexa. Parasitology. 2015;142(Suppl 1):S57-70.

2. Jensen AR, Adams Y, Hviid L. Cerebral Plasmodium falciparum malaria: the role of PfEMP1 in its pathogenesis and immunity, and PfEMP1-based vaccines to prevent it. Immunol Rev. 2020;293(1):230-52.

3. Otto TD, Bohme U, Sanders M, Reid A, Bruske El, Duffy CW, Bull PC, Pearson RD, Abdi A, Dimonte S, et al. Long read assemblies of geographically dispersed Plasmodium falciparum isolates reveal highly structured subtelomeres. Wellcome Open Res. 2018;3:52.

4. RaskTS, Hansen DA, Theander TG, Gorm Pedersen A, Lavstsen T. Plasmodium falciparum erythrocyte membrane protein 1 diversity in seven genomes--divide and conquer. PLoS Comput Biol. 2010;6(9).

5. Smith JD, Rowe JA, Higgins MK, Lavstsen T. Malaria's deadly grip: cytoadhesion of Plasmodium falciparum-infected erythrocytes. Cell Microbiol. 2013;15(12):1976-83.

6. Otto TD, Assefa SA, Böhme U, Sanders M, Kwiatkowski D, Pf3k consortium, Berriman M, Newbold C. Evolutionary analysis of the most polymorphic gene family in falciparum malaria. Wellcome Open Res. 2019;4(193).

7. Smith JD. The role of PfEMP1 adhesion domain classification in Plasmodium falciparum pathogenesis research. Mol Biochem Parasitol. 2014;195(2):82-7.

8. Larremore DB, Sundararaman SA, Liu W, Proto WR, Clauset A, Loy DE, Speede S, Plenderleith LJ, Sharp PM, Hahn BH, et al. Ape parasite origins of human malaria virulence genes. Nat Commun. 2015;6:8368.

9. Andrade CM, Fleckenstein H, Thomson-Luque R, Doumbo S, Lima NF, Anderson C, Hibbert J, Hopp CS, Tran TM, Li S et al. Increased circulation time of Plasmodium falciparum underlies persistent asymptomatic infection in the dry season. Nat Med. 2020.

10. Bull PC, Berriman M, Kyes S, Quail MA, Hall N, Kortok MM, Marsh K, Newbold Cl. Plasmodium falciparum variant surface antigen expression patterns during malaria. PLoS Pathog. 2005;1 (3):e26.

11. Jespersen JS, Wang CW, Mkumbaye SI, Minja DT, Petersen B, Turner L, Petersen JE, Lusingu JP, Theander TG, Lavstsen T. Plasmodium falciparum var genes expressed in children with severe malaria encode CIDRalpha1 domains. EMBO Mol Med. 2016;8(8):839-50.

12. Kyriacou HM, Stone GN, Challis RJ, Raza A, Lyke KE, Thera MA, Kone AK, Doumbo OK, Plowe CV, Rowe JA. Differential var gene transcription in Plasmodium falciparum isolates from patients with cerebral malaria compared to hyperparasitaemia. Mol Biochem Parasitol. 2006;150(2):211-8.

13. Kessler A, Dankwa S, Bernabeu M, Harawa V, Danziger SA, Duffy F, Kampondeni SD, Potchen MJ, Dambrauskas N, Vigdorovich $V$ et al. Linking EPCR-binding PfEMP1 to brain swelling in pediatric cerebral malaria. Cell Host Microbe. 2017;22(5):601-614.

14. Zilversmit MM, Chase EK, Chen DS, Awadalla P, Day KP, McVean G. Hypervariable antigen genes in malaria have ancient roots. BMC Evol Biol. 2013;13:110.

15. Lavstsen T, Turner L, Saguti F, Magistrado P, Rask TS, Jespersen JS, Wang CW, Berger SS, Baraka V, Marquard AM, et al. Plasmodium falciparum erythrocyte membrane protein 1 domain cassettes 8 and 13 are associated with severe malaria in children. Proc Natl Acad Sci USA. 2012;109(26):E1791-1800.

16. Carrington E, Otto TD, Szestak T, Lennartz F, Higgins MK, Newbold Cl, Craig AG. In silico guided reconstruction and analysis of ICAM-1-binding var genes from Plasmodium falciparum. Sci Rep. 2018;8(1):3282.

17. Wichers JS, Tonkin-Hill G, Thye T, Krumkamp R, Kreuels B, Strauss J, von Thien H, Scholz JA, Smedegaard Hansson H, Weisel Jensen $R$ et al. Common virulence gene expression in adult first-time infected malaria patients and severe cases. Elife. 2021;10.

18. Higgins MK, Carrington M. Sequence variation and structural conservation allows development of novel function and immune evasion in parasite surface protein families. Protein Sci. 2014;23(4):354-65.

\section{Publisher's Note}

Springer Nature remains neutral with regard to jurisdictional claims in published maps and institutional affiliations.

Ready to submit your research? Choose BMC and benefit from:

- fast, convenient online submission

- thorough peer review by experienced researchers in your field

- rapid publication on acceptance

- support for research data, including large and complex data types

- gold Open Access which fosters wider collaboration and increased citations

- maximum visibility for your research: over $100 \mathrm{M}$ website views per year

At BMC, research is always in progress.

Learn more biomedcentral.com/submissions 